\title{
B7-H3 promotes aerobic glycolysis and chemoresistance in colorectal cancer cells by regulating HK2
}

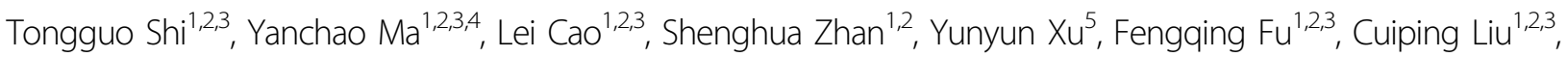 \\ Guangbo Zhang ${ }^{1,2,3}$, Zhenxin Wang ${ }^{1,2,3}$, Ruoqin Wang ${ }^{1,2,3}$, Huimin Lu ${ }^{1,2,3}$, Binfeng Lu' ${ }^{6}$, Weichang Chen ${ }^{1,2,3,4}$ and \\ Xueguang Zhang ${ }^{1,2,3}$
}

\begin{abstract}
Accumulating evidence suggests that aerobic glycolysis is important for colorectal cancer (CRC) development. However, the underlying mechanisms have yet to be elucidated. B7-H3, an immunoregulatory protein, is broadly overexpressed by multiple tumor types and plays a vital role in tumor progression. In this study, we found that overexpression of $\mathrm{B} 7-\mathrm{H} 3$ effectively increased the rate of glucose consumption and lactate production, whereas knockdown of B7-H3 had the opposite effect. Furthermore, we showed that B7-H3 increased glucose consumption and lactate production by promoting hexokinase 2 (HK2) expression in CRC cells, and we also found that HK2 was a key mediator of B7-H3-induced CRC chemoresistance. Depletion of HK2 expression or treating cells with HK2 inhibitors could reverse the $\mathrm{B} 7-\mathrm{H} 3$-induced increase in aerobic glycolysis and $\mathrm{B} 7-\mathrm{H} 3$-endowed chemoresistance of cancer cells. Moreover, we verified a positive correlation between the expression of B7-H3 and $\mathrm{HK} 2$ in tumor tissues of CRC patients. Collectively, our findings suggest that $\mathrm{B} 7-\mathrm{H} 3$ may be a novel regulator of glucose metabolism and chemoresistance via controlling HK2 expression in CRC cells, a result that could help develop B7-H3 as a promising therapeutic target for CRC treatment.
\end{abstract}

\section{Introduction}

Colorectal cancer (CRC) is the third most prevalent cancer type in the world ${ }^{1}$. Although screening and radical surgical resection have significantly improved the 5-year survival rate of patients with CRC in the early stage, the majority of patients are diagnosed at advanced stages ${ }^{2}$. Unfortunately, very few therapy options are currently available for effective treatment of advanced CRC. Therefore, it is imperative to understand the molecular

\footnotetext{
Correspondence: Weichang Chen (weichangchen@126.com) or

Xueguang Zhang (xueguangzh@126.com)

'Jiangsu Institute of Clinical Immunology, The First Affiliated Hospital of Soochow University, 708 Renmin Road, Suzhou, China

2Jiangsu Key Laboratory of Clinical Immunology, Soochow University, 708 Renmin Road, Suzhou, China

Full list of author information is available at the end of the article.

These authors contributed equally: Tongguo Shi, Yanchao Ma, Lei Cao

Edited by A. Finazzi-Agrò
}

mechanisms underlying CRC progression and to identify precise and effective biomarkers.

B7-H3, also known as CD276, is an important immune checkpoint member of the B7-CD28 family ${ }^{3}$. As a type I transmembrane protein, two $\mathrm{B} 7-\mathrm{H} 3$ isoforms (4IgB7-H3 and 2Ig-B7-H3) have been identified; 4Ig-B7-H3 is the main isoform in humans and 2Ig-B7-H3 is the only isoform in mice ${ }^{4}$. Because of the lack of an identified receptor, the immunologic function of $\mathrm{B} 7-\mathrm{H} 3$ remains controversial, with conflicting costimulatory and coinhibitory functions ${ }^{5}$. However, B7-H3 has been reported to be a pivotal non-immunologically multifunctional protein involved in the regulation of many key cellular events. Interestingly, accumulated evidence indicates that aberrant expression of $\mathrm{B} 7-\mathrm{H} 3$ is a common characteristic of CRC and is consistently correlated with poor patient prognosis, suggesting its emerging importance in CRC

\section{(c) The Author(s) 2019}

(c) (i) Open Access This article is licensed under a Creative Commons Attribution 4.0 International License, which permits use, sharing, adaptation, distribution and reproduction cc) in any medium or format, as long as you give appropriate credit to the original author(s) and the source, provide a link to the Creative Commons license, and indicate if changes were made. The images or other third party material in this article are included in the article's Creative Commons license, unless indicated otherwise in a credit line to the material. If material is not included in the article's Creative Commons license and your intended use is not permitted by statutory regulation or exceeds the permitted use, you will need to obtain permission directly from the copyright holder. To view a copy of this license, visit http://creativecommons.org/licenses/by/4.0/. 
progression $^{6,7}$. A previous report showed that B7-H3 could promote epithelial to mesenchymal transition (EMT) in CRC cells, evidenced by decreasing the expression of E-cadherin and $\beta$-catenin and up-regulating $\mathrm{N}$-cadherin and vimentin expression ${ }^{8}$. In addition, the administration of anti-B7-H3-drug conjugates to various human CRC xenografts could simultaneously destroy both tumor cells and tumor vasculature ${ }^{9}$. Moreover, B7H3 could upregulate BRCA1/BRCA2-containing complex subunit 3 (BRCC3) or X-ray repair cross complementing group 1 (XRCC1) expression to antagonize DNA damage caused by 5-fluorouracil (5-FU) or oxaliplatin $(\mathrm{L}-\mathrm{OHP})^{10,11}$. Although those studies have suggested multiple roles for $\mathrm{B} 7-\mathrm{H} 3$ in $\mathrm{CRC}$, it is necessary to understand the exact roles of $\mathrm{B} 7-\mathrm{H} 3$ in the development and progression of CRC.

Cancer cell metabolism is characterized by an increase in glycolysis and lactate production even in the presence of abundant oxygen, known as the Warburg effect or aerobic glycolysis ${ }^{12}$. Aerobic glycolysis confers on cancer cells a growth advantage by providing energy and biosynthesis building blocks ${ }^{13}$. It has been widely accepted that aerobic glycolysis is a distinctive hallmark of cancer, and antitumor therapeutic agents targeting aerobic glycolysis are being developed ${ }^{14}$. Accumulated evidence has indicated that oncogenic alterations may participate in the regulation of aerobic glycolysis ${ }^{15}$. For instance, hypoxiainducible factor $1 \alpha$ (HIF-1 $\alpha)$, which is rapidly upregulated under hypoxic conditions, increases the expression of glycolysis-associated proteins, such as glucose transporters and glycolytic enzymes ${ }^{16}$. Another important oncogenic protein, c-MYC, was reported to promote glycolysis through transactivating the glycolytic enzyme genes ${ }^{17}$. Oncogenic signaling pathways, such as the PI3K/Akt,

STAT3 and Wnt/ $\beta$-catenin pathways, are also known as regulators of cancer cell metabolism ${ }^{18-20}$. In addition, miRNA-mediated posttranscriptional regulation is involved in regulating cancer aerobic glycolysis ${ }^{21}$. These studies suggest that aerobic glycolysis in cancer cells is far more complicated than expected and warrants further investigation.

Despite a promoting aerobic glycolysis of B7-H3 in breast cancer, evidenced by increasing glucose uptake and lactate production ${ }^{22,23}$, the effects of $\mathrm{B} 7-\mathrm{H} 3$ on aerobic glycolysis in CRC remain largely unknown. In this study, we investigated whether and how B7-H3 modulated glucose metabolism in CRC. We showed here that B7-H3 enhanced aerobic glycolysis by upregulating the expression of the glycolytic enzyme hexokinase 2 (HK2), a key mediator of aerobic glycolysis, in CRC cells. B7-H3 promoted HK2 expression by activating STAT3 signaling. Importantly, we demonstrated that HK2 expression was critical for B7-H3-mediated CRC chemoresistence both in vitro and in vivo. These findings revealed a previously unrecognized mechanism of B7-H3 in human CRC by affecting the aerobic glycolysis and chemoresistence through regulation of HK2.

\section{Results \\ B7-H3 promotes glucose consumption and lactate production in colorectal cancer cells}

To determine the effects of $\mathrm{B} 7-\mathrm{H} 3$ on glucose consumption and lactate production in colorectal cancer cells, we established two CRC cell lines stably expressing B7-H3, with $\mathrm{B} 7-\mathrm{H} 3$ protein levels more than twice as high as that of control cells (Fig. 1a). Both glucose consumption and lactate production were significantly increased in B7-H3overexpression HCT116 and RKO cells compared with control cells without B7-H3 overexpression (Fig. 1b, c). On the other hand, we knocked down the expression of B7-H3 in HCT116 and RKO cells using two independent siRNAs specific for B7-H3 (B7-H3 siRNA-1 and B7-H3 siRNA-2), which reduced the expression of $\mathrm{B} 7-\mathrm{H} 3$ to less than $70 \%$ of that of the scrambled control (Fig. 1d). Reduction of B7H3 significantly decreased glucose consumption and lactate production in both cell lines (Fig. 1e, f). Collectively, these data demonstrate that $\mathrm{B} 7-\mathrm{H} 3$ promoted aerobic glycolysis and lactate production in CRC cells.

\section{B7-H3 controls expression of HK2 via STAT3}

To investigate how B7-H3 regulates aerobic glycolysis in $\mathrm{CRC}$, we analyzed the expression of a spectrum of key glycolysis-related genes, including Glucose transporter 1 (GLUT1), Glucose transporter 4 (GLUT4), Lactate dehydrogenase A (LDHA), Lactate dehydrogenase B (LDHB), HK2, pyruvate kinase M 2 (PKM2), HIF- $1 \alpha$ and pyruvate dehydrogenase kinase 1 (PDK1) in B7-H3overexpressing HCT116, and RKO cells with real-time quantitative PCR (RT-qPCR) (Fig. 2a, b). We found that the expression level of HK2, a known mediator of aerobic glycolysis, was the most increased in both HCT116 and RKO cells with overexpression of B7-H3 (Fig. 2a, b). In contrast, knockdown of B7-H3 significantly reduced the mRNA and protein levels of HK2 in HCT116 and RKO cells (Fig. 2c, d). In addition, western blot analysis revealed that knockdown of B7-H3 significantly reduced the protein levels of HK2 in HCT116 and RKO cells (Fig. 2e), while overexpression of $\mathrm{B} 7-\mathrm{H} 3$ resulted in a significant increase in HK2 protein expression (Fig. 2f). Thus, our results suggest that B7-H3 positively regulated HK2 expression.

We next sought to explore the signaling pathways by which B7-H3 mediated the expression of HK2. Previous studies have shown that the expression of HK2 could be regulated by STAT3 in tumor cells such as MCF7, HepG2, and A549 cells $^{24,25}$. In addition, STAT3 signaling is known to be a downstream target of $\mathrm{B} 7-\mathrm{H} 3$ in $\mathrm{CRC}^{26,27}$. We hypothesized that the upregulation of HK2 in both 
a

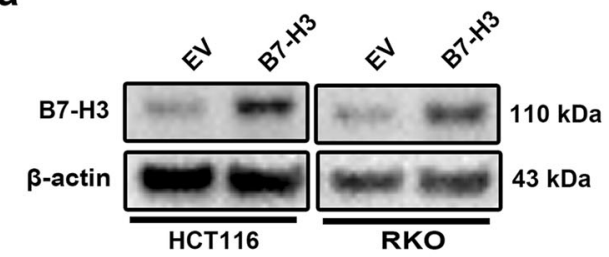

b
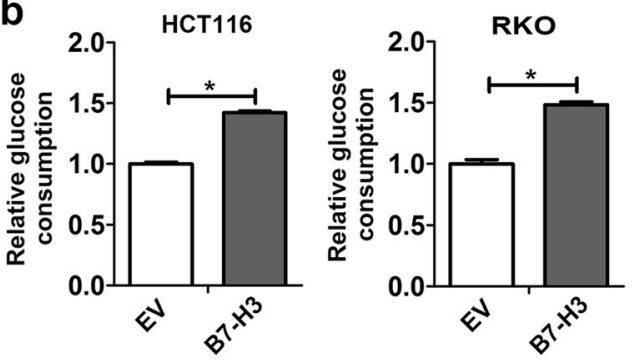

C
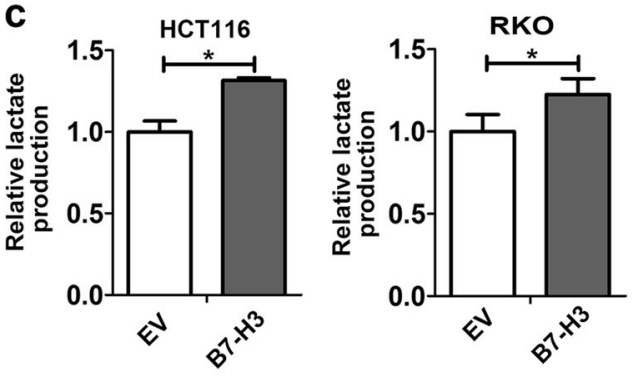

d

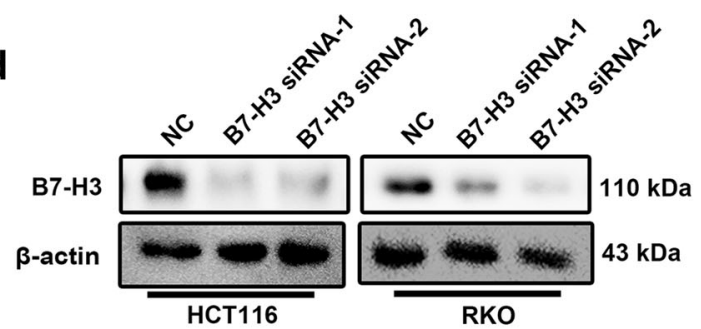

e
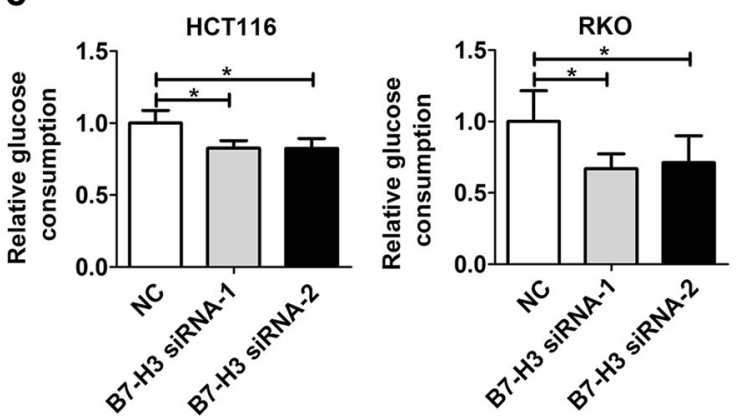

f

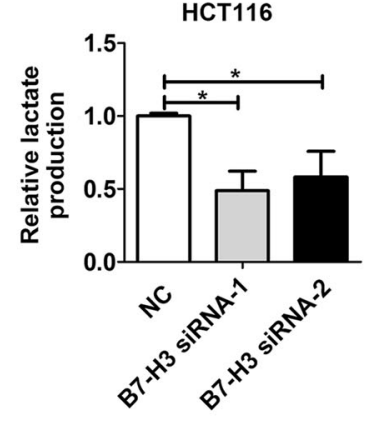

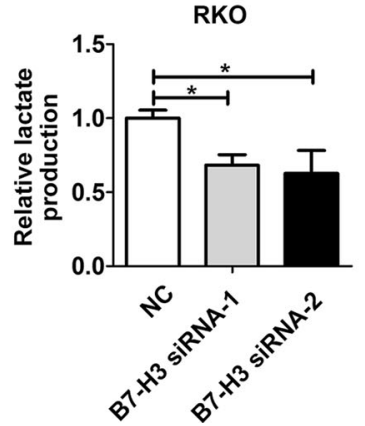

Fig. 1 B7-H3 promoted aerobic glycolysis in CRC cells. a B7-H3 protein levels in both B7-H3-overexpressing HCT116 and RKO cells were analyzed by western blot. $\beta$-actin served as a loading control. b, c Glucose consumption (b) and lactate production (c) were measured in both B7-H3overexpressing HCT116 and RKO cells. d B7-H3 protein levels in both HCT116 and RKO cells were analyzed by western blot after transfection with siRNA negative control (NC), B7-H3 siRNA-1 or B7-H3 siRNA-2. $\beta$-actin served as a loading control. e, f Glucose consumption (e) and lactate production (f) were measured in both HCT116 and RKO cells transfected with NC, B7-H3 siRNA-1 or B7-H3 siRNA-2. Values were expressed as means (SEMs). Five samples were analyzed per condition, and the experiments were performed in triplicate. ${ }^{*} P<0.05$

B7-H3-overexpressing CRC cells might be STAT3 dependent. Indeed, B7-H3 siRNA treatment reduced the activity of STAT3 (Fig. 2e). Conversely, increased STAT3 activity was observed in both B7-H3-overexpressing HCT116 and RKO cells (Fig. 2f). Moreover, we observed that cryptotanshinone, a STAT3 phosphorylation inhibitor, that blocked the phosphorylation of STAT3, significantly decreased the expression of HK2 in both B7H3-overexpressing HCT116 and RKO cells (Fig. 2f). These results suggest that $\mathrm{B} 7-\mathrm{H} 3$ could regulate the expression of HK2 by the STAT3 signaling pathway in CRC cells (Fig. 2g).

\section{B7-H3 promotes aerobic glycolysis via HK2}

When HK2 was downregulated by HK2 siRNA, B7-H3induced increase in glucose uptake and lactate production was obviously attenuated (Fig. 3a-c). Additionally, 2Deoxy-D-glucose (2-DG), a HK2 inhibitor, was used to treat B7-H3-overexpressing HCT116 and RKO cells. Treatment with 2-DG abolished the B7-H3-induced increase in glucose uptake and lactate production (Fig. 3d, e). These results indicate that B7-H3-induced aerobic glycolysis was HK2 dependent.

\section{B7-H3 increases CRC cell chemoresistance via HK2 in vitro}

In addition to promoting tumorigenesis, aerobic glycolysis of cancer cells provides an environment that often increases drug resistance ${ }^{28}$. It has been shown that knockdown of HK2 can sensitize glioblastoma multiforme cells to therapeutic drugs ${ }^{29}$. Here, we chose $25 \mathrm{nM}$ HK2 siRNA or $5 \mathrm{mM} 2-\mathrm{DG}$, a concentration resulting in around $10 \%$ cells viablility in both HCT116 and RKO 

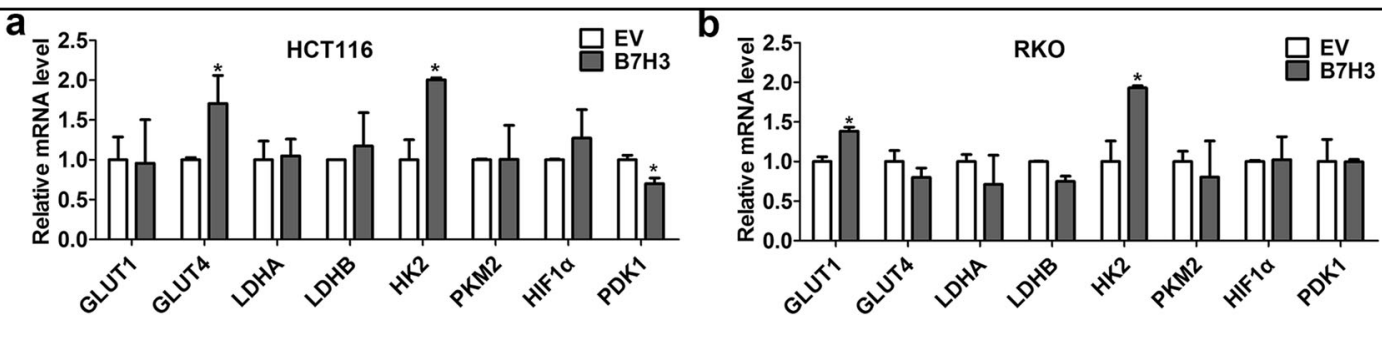

C

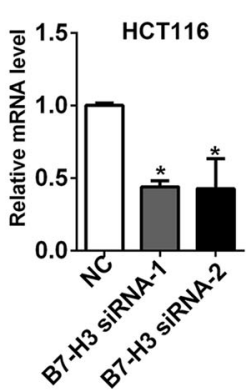

d

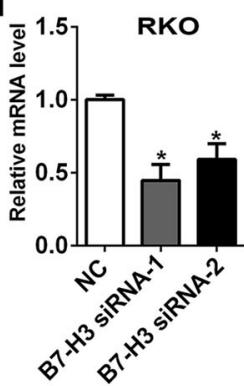

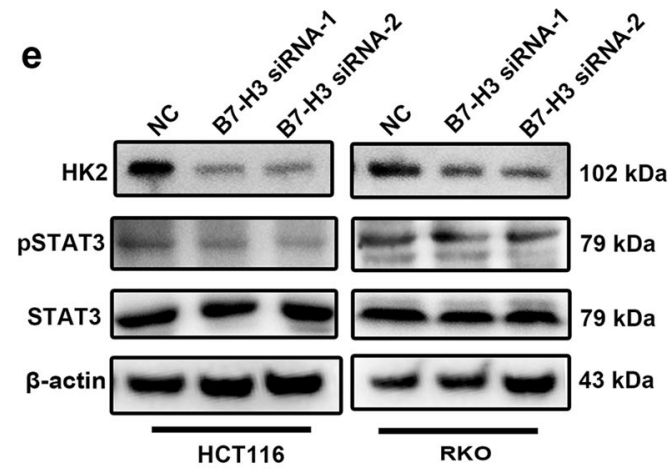

g

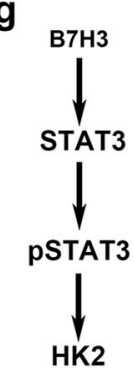

Fig. 2 B7-H3 promoted the expression of HK2 in CRC cells. $\mathbf{a}$, b The expression of glycolysis-related genes was detected by RT-qPCR in both B7H3-overexpressing HCT116 (a) and RKO (b) cells. c, d The mRNA level of HK2 was detected by RT-qPCR in both HCT116 (c) and RKO (d) cells after transfection with siRNA NC, B7-H3 siRNA-1 or B7-H3 siRNA-2. e HK2 protein level and STAT3 activation (examined by the p-STAT3 expression level) were detected by western blot in both $\mathrm{HCT} 116$ and RKO cells after transfection with NC, B7-H3 siRNA-1 or B7-H3 siRNA-2. $\beta$-actin served as a loading control. $\mathbf{f}$ HK2 protein level and STAT3 activation (examined by the p-STAT3 expression level) were detected by western blot in both B7-H3overexpressing HCT116 and RKO cells. $\beta$-actin served as a loading control. g Schematic representation of the proposed B7-H3/STAT3/HK2 axis. Values are expressed as means (SEMs). Five samples were analyzed per condition, and the experiments were performed in triplicate. ${ }^{*} P<0.05$

cells, to evaluate whether HK2 was critical for B7-H3regulated cancer chemoresistance.

The effect of B7-H3 on CRC cell chemoresistance was determined with clonogenic assay. As shown in Fig. 4a, b, B7-H3 overexpression significantly enhanced the clonogenic potential of HCT116 and RKO cells upon exposure to L-OHP. In addition, cell viability analysis showed that B7-H3 enhanced L-OHP resistance of HCT116 and RKO cells (Fig. 4c). These results were further confirmed by treatment with another chemotherapy drug, 5-FU (Fig. 4d). Furthermore, flow cytometry analysis showed less apoptosis in B7-H3overexpressing HCT116 and RKO cells than in control cells treated with L-OHP (Fig. 4e and Supplemental Fig. S1). Finally, enhanced B cell lymphoma 2 (Bcl-2) expression and reduced $\mathrm{Bcl}-2$-Associated $\mathrm{X}$ (Bax) expression was observed in B7-H3-overexpressing HCT116 and RKO cells that were treated with L-OHP (Fig. 4f).

To examine whether the CRC cell resistance to chemotherapeutic drugs by $\mathrm{B} 7-\mathrm{H} 3$ overexpression occurs through elevated glucose metabolism, HK2 siRNA or 2DG was used to treat B7-H3-overexpressing HCT116 and RKO cells. We found that chemoresistance of B7-H3overexpressing HCT116 and RKO cells to L-OHP and 5FU was reversed by HK2 siRNA or 2-DG (Fig. 4).

\section{$\mathrm{B} 7-\mathrm{H} 3 / \mathrm{HK} 2$ pathway induces CRC chemoresistance in vivo}

To confirm the chemoresistance effect of $\mathrm{B} 7-\mathrm{H} 3$ on $\mathrm{CRC}$ in vivo, xenograft models of B7-H3-overexpressing 


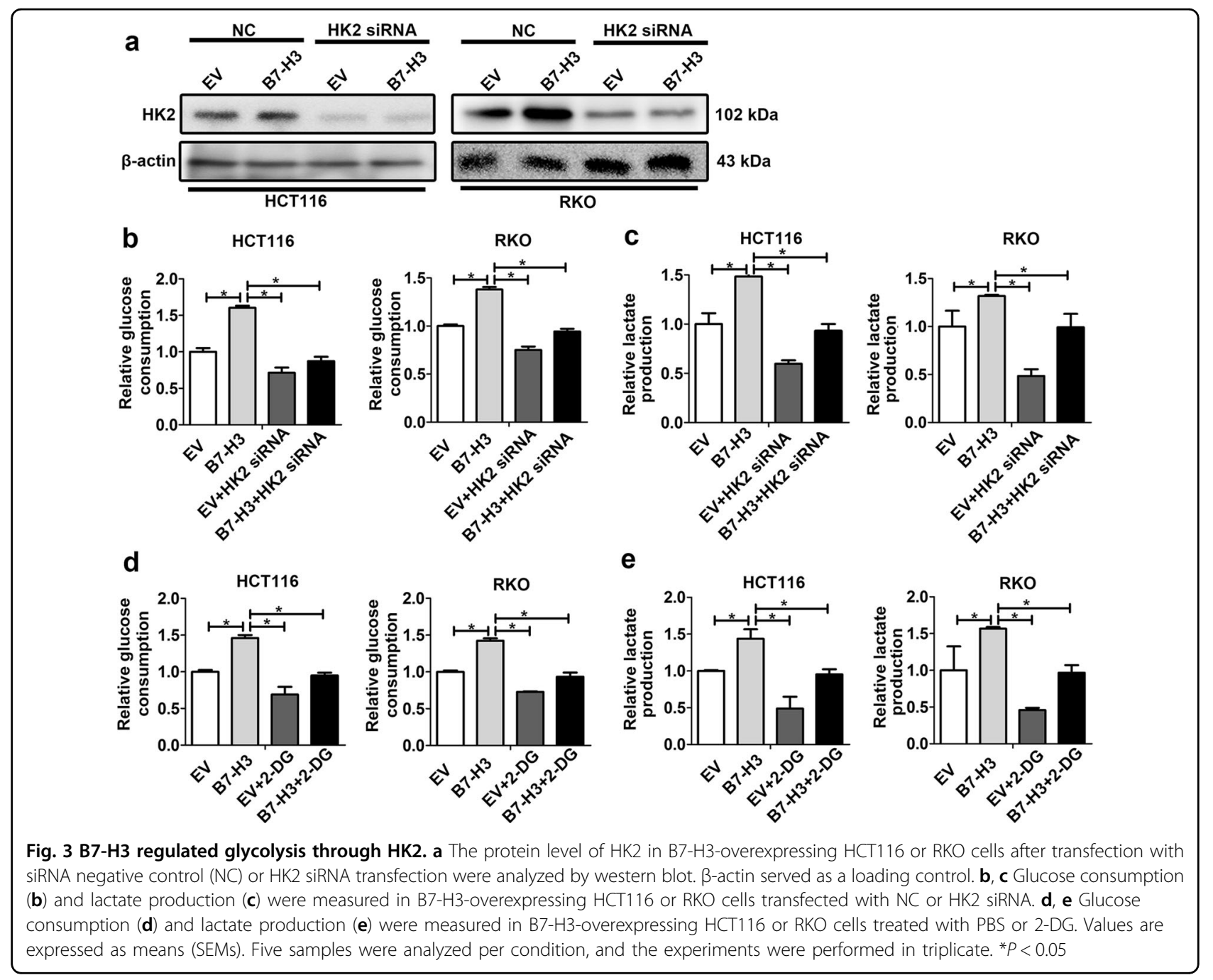

HCT116 tumors or control tumors in nude mice were established, and L-OHP was administered at different timepoints (Fig. 5a). The tumor sizes, images and mass revealed that $\mathrm{B} 7-\mathrm{H} 3$ had no effect on untreated HCT116 tumor growth (Fig. 5b-d). However, tumors that were derived from B7-H3-overexpressing HCT116 cells were significantly more resistant to L-OHP than did tumors derived from control vector HCT116 cells (Fig. 5b-d). We next confirmed whether B7-H3regulated CRC chemoresistance was HK2 dependent in vivo. $\mathrm{L}-\mathrm{OHP}$ and $2-\mathrm{DG}$ were administered to the mice injected with B7-H3-overexpressing HCT116 cells at different timepoints (Fig. 5e). We found that 2DG significantly reversed the resistance of $\mathrm{B} 7-\mathrm{H} 3-$ overexpressing HCT116 tumors to L-OHP in vivo (Fig. 5f-h). The data suggested that B7-H3-induced glycolysis promoted CRC cell chemoresistance, and this was reversed by glycolysis inhibition with 2-DG treatment in vivo.

\section{B7-H3 protein levels positively correlate with HK2} expression in CRC patient tumor tissue specimens

Previous studies suggested that B7-H3 was aberrantly expressed in colorectal cancer and consistently correlated with poor colon cancer patient outcomes ${ }^{8,30}$. To investigate the clinical correlation between B7-H3 and HK2 protein levels in CRC patient specimens, we analyzed 126 pairs of the primary tumor lesions and corresponding normal adjacent tissues in Chinese patients with CRC. IHC analysis showed that both $\mathrm{B} 7-\mathrm{H} 3$ and $\mathrm{HK} 2$ proteins were significantly upregulated in CRC samples compared with normal adjacent tissues (Fig. 6a-c). Moreover, HK2 was positively correlated with $\mathrm{B} 7-\mathrm{H} 3$ expression in this cohort (Fig. 6d). Additionally, both B7-H3 and HK2 expression increased with tumor stage. The levels of B7$\mathrm{H} 3$ and HK2 were higher in advanced clinical stages (III and IV) than in early stages (I and II) (Fig. 6e, f). Thus, the levels of B7-H3 and HK2 were positively correlated in human CRC specimens. 


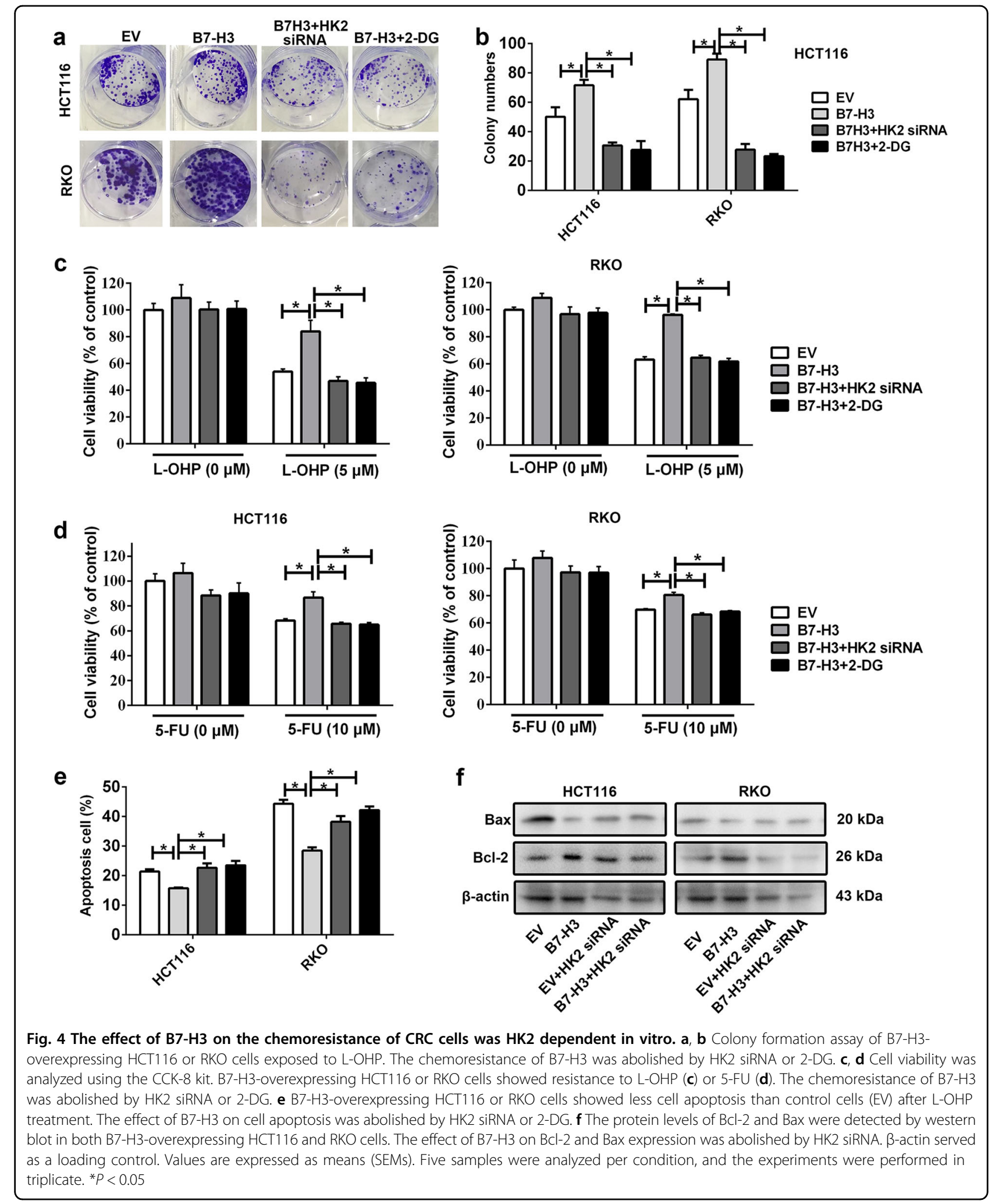

\section{Discussion}

In this study, we focused our research on the regulation of CRC aerobic glycolysis and chemoresistance by B7-H3.
Although it has been reported that $\mathrm{B} 7-\mathrm{H} 3$ can regulate aerobic glycolysis in breast cancer, the underlying molecular mechanism of $\mathrm{B} 7-\mathrm{H} 3$ in the regulation of aerobic 

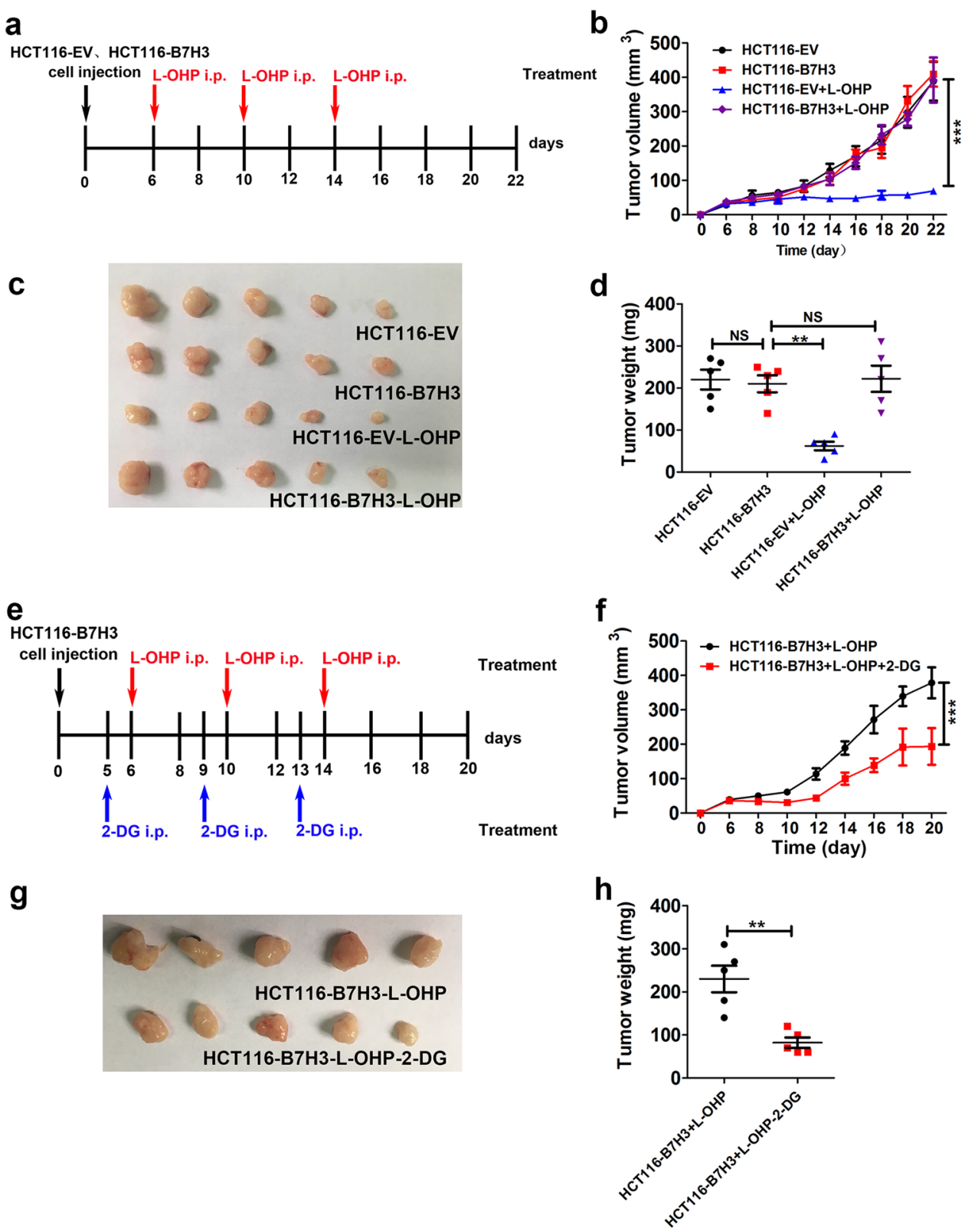

Fig. 5 The effect of B7-H3 on the chemoresistance of CRC cells was HK2 dependent in vivo. a Schematic overview of the xenograft tumor model and L-OHP treatment. $\mathbf{b}$ The volumes of the B7-H3-overexpressing HCT116 tumors in nude mice following L-OHP treatment. c A representative image of B7-H3-overexpressing HCT116 tumors in nude mice following L-OHP treatment. $\mathbf{d}$ The weights of the B7-H3-overexpressing HCT116 tumors in nude mice following L-OHP treatment. e Schematic overview of the xenograft tumor model and 2-DG or L-OHP treatment. f The volumes of the B7-H3-overexpressing HCT116 tumors in nude mice following 2-DG and L-OHP treatment. $\mathbf{g}$ A representative image of the overexpressing B7-H3 HCT116 tumors in nude mice following 2-DG and L-OHP treatment. $\mathbf{h}$ The weights of the B7-H3-overexpressing HCT116 tumors in nude mice following 2-DG and L-OHP treatment. Values are expressed as means (SEMs). $n=5$ mice per group. ${ }^{*} P<0.05$

glycolysis in CRC remains unknown ${ }^{22,23}$. Here, we showed that B7-H3 promoted glucose uptake and lactate production in CRC cells. Considering the aberrant expression of $\mathrm{B} 7-\mathrm{H} 3$ in $\mathrm{CRC}^{6,7}$ (Fig. 6a) and the emerging importance of aerobic glycolysis for cancer development, we therefore conclude that $\mathrm{B} 7-\mathrm{H} 3$ may play a critical role in the regulation of glucose metabolism in CRC cells.
Previous studies showed that HIF- $1 \alpha$ played key roles in regulating aerobic glycolysis under hypoxic conditions $^{16,31}$. It has also been shown that B7-H3 could regulate aerobic glycolysis via HIF- $1 \alpha$ in breast cancer cells ${ }^{22}$. However, we observed that the protein level of HIF-1 $\alpha$ was only marginally affected by B7-H3 (Supplemental Fig. S2), suggesting exclusion of HIF-1 $\alpha$ involvement. 


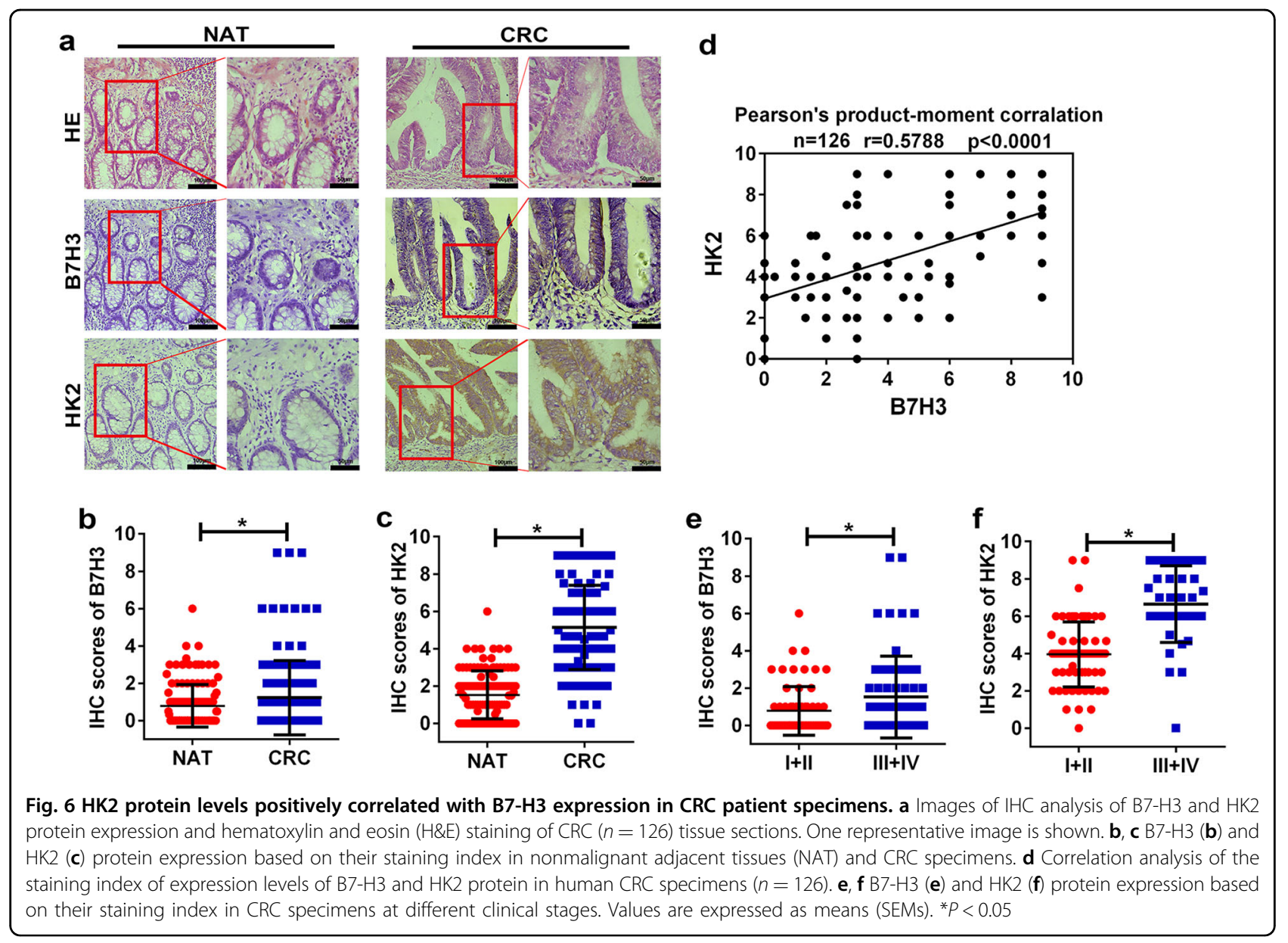

Moreover, given that the basal protein level of HIF-1 $\alpha$ is very low under normoxia, it is inexplicable why cancer cells adapt to glycolysis, which is less efficient, for energy sources even in the presence of a rich oxygen supply ${ }^{21}$. In addition, Nunesxavier et al. reported that B7-H3 overexpression did not alter activation of the Akt/mTOR pathway but that the high $\mathrm{B} 7-\mathrm{H} 3$ expression levels in tumor cells increased cell survival and glycolysis ${ }^{23}$. Therefore, we surmised that other mechanisms might be involved in B7-H3-mediated aerobic glycolysis in CRC cells. Indeed, we found that the mRNA and protein levels of HK2 in CRC cells could be modulated by $\mathrm{B} 7-\mathrm{H} 3$ (Fig. 2).

Until now, hexokinases (HKs) are tissue-specific isoenzymes that catalyze the first step of glucose metabolism by phosphorylating glucose to glucose- 6 -phosphatase ${ }^{32}$. The mammalian HK family has four isoforms, HK1, 2, 3, and 4. Among these isoforms, HK2 is critically important for aerobic glycolysis in multiple cancer types, including glioblastoma multiforme, breast and ovarian cancer ${ }^{29,33,34}$. Overexpression of $\mathrm{HK} 2$ is also observed in multiple tumor tissues and is associated with poor prognosis in tumor patients $^{35}$. 2-DG, a well-known HK2 inhibitor, induces cancer cell death by depleting intracellular glucose levels ${ }^{36}$. Therefore, HK2 is regarded as a key player in aerobic glycolysis and has been proposed as a therapeutic target for cancers ${ }^{37}$. The expression of HK2 in cancer cells could be regulated at the transcriptional and posttranscriptional level. Previous studies showed that HK2 was directly regulated by miR-199a-5p in liver cancer ${ }^{38}$; by miR-155 in lung cancer ${ }^{39}$; and by miR-143 in colon cancers $^{40}$. Zhuo et al. revealed that PI3K/Akt signaling inhibited cell apoptosis and promoted tumor growth by upregulating HK2 expression in pediatric osteosarcoma ${ }^{41}$. Additionally, STAT3 signaling has been shown to accelerate aerobic glycolysis by upregulating HK2 in bladder, breast and hepatocellular carcinoma ${ }^{24,42,43}$. In this study, we found that B7-H3 could activate STAT3 and then upregulate HK2 expression in CRC cells (Fig. 2e, f). Importantly, cryptotanshinone could inhibit the expression of HK2 induction by B7-H3 in CRC cells (Fig. 2f). These results suggested that $\mathrm{B} 7-\mathrm{H} 3$ could regulate the expression of HK2 via the STAT3 signaling pathway (Fig. 2g).

Mounting evidence suggests that $\mathrm{B} 7-\mathrm{H} 3$ is intimately associated with chemotherapeutic resistance. For 
instance, B7-H3 silencing increased the sensitivity of Maver and Z138 cells to rituximab and bendamustine and enhanced drug-induced apoptosis in mantle cell lymphoma ${ }^{44}$. ShRNA targeting B7-H3 significantly enhanced the sensitivity of U937 cells to first-line chemotherapy drugs (idarubicin and cytarabine) used in acute monocytic leukemia ${ }^{45}$. Upregulation of B7-H3 diminished the sensitization role of astragaloside IV, a component of Traditional Chinese Medicine Astragalus membranaceus, in cellular responses to cisplatin in non-small cell lung cancer $^{46}$. Moreover, overexpression of B7-H3 could affect the efficacy of DNA damage-inducing anticancer drugs in $\mathrm{CRC}^{10,11}$. Herein, we showed novel relationships between B7-H3-mediated aerobic glycolysis and chemoresistance. Previous studies have demonstrated that the expression levels of HK2, PKM2, LDHA, and GLUT1 are correlated with the development of chemoresistance in malignant tumors ${ }^{47}$. Glycolytic inhibitors, such as 3-bromopyruvate and 2-DG, could enhance the sensitivity of tumor cells to chemotherapy drugs ${ }^{48,49}$. In the present study, we found that inhibition of aerobic glycolysis by 2-DG or knockdown of HK2 expression effectively abolished B7-H3 overexpression-induced resistance to $\mathrm{L}-\mathrm{OHP}$ and 5 -FU in vitro and in vivo (Figs. 4 and 5). Our findings revealed that B7-H3-mediated aerobic glycolysis played a critical role in the development of chemoresistance in CRC cells and suggested that $\mathrm{B} 7-\mathrm{H} 3$ might be a potential target to prevent the development of CRC chemoresistance.

In sum, we investigated the function of B7-H3 in CRC aerobic glycolysis and elucidated an important molecular mechanism of B7-H3 in the development of CRC chemoresistance. We revealed that B7-H3 mediated the activation of STAT3 and subsequent expression of HK2, to promote glycolysis in CRC cells. Moreover, B7H3-mediated aerobic glycolysis could enhance the chemoresistance of CRC cells in vitro and in vivo. Therefore, our findings have uncovered that $\mathrm{B} 7-\mathrm{H} 3$ is a novel regulatory factor of glucose metabolism and chemoresistance via controlling HK2 expression in CRC cells, suggesting $\mathrm{B} 7-\mathrm{H} 3$ as a promising therapeutic target for CRC treatment.

\section{Materilas and methods}

Cell culture, cell lentivirus infection, and cell transfection

HCT116 and RKO cells (ATCC, Manassas, Virginia, USA) were cultured in Dulbecco's modified Eagle medium (DMEM) (Invitrogen, Carlsbad, CA) containing 10\% fetal bovine serum (FBS) (Invitrogen) at $37^{\circ} \mathrm{C}$ in a humidified atmosphere of $5 \% \mathrm{CO}_{2}$.

Lentivirus vector carrying human 4IgB7-H3 cDNA was generated by Genechem Co., Ltd (Shanghai, China). An empty backbone vector was used as a control. For lentivirus infection, HCT116 cells or RKO cells were grown to $30 \%$ confluence in 6-well plates and were infected with lentiviral particles at a multiplicity of infection (MOI) of 20. The infection efficiency was confirmed by counting GFP-expressing cells under fluorescence microscopy $72 \mathrm{~h}$ after infection.

Human B7-H3 siRNA-1 (5'-GCUGUCUGUCUGUCUCAUUTT-3'), human B7-H3 siRNA-2 (5'-GUGCUGGAGAAAGAUCAAATT-3'), human HK2 siRNA (5'-CACGATGAAATTGAACCTGGT- $3^{\prime}$ ) and their control siRNA were purchased from GenePharma Co. Ltd. (Suzhou, China). HCT116 or RKO cells were transfected with siRNA reagents using Lipofectamine 2000 (Invitrogen) according to the manufacturer's instructions. Transfection efficiency was determined by RT-qPCR and western blot.

\section{Total RNA isolation and RT-qPCR assays}

Total RNA from cultured cells was prepared using TRIzol reagent (Invitrogen) according to the manufacturer's instructions. For mRNA detection, RT-qPCR assays were performed on a CFX96 Touch $^{\mathrm{TM}}$ Real-Time PCR system (Bio-Rad, CA, USA) using EvaGreen Dye (Biotium, Hayward, CA, USA). For analysis of individual genes, $0.5 \mu \mathrm{g}$ of total RNA was used for DNA synthesis and RT-qPCR was conducted using a SYBR PrimeScript RT-qPCR Kit (Takara Bio, Shiga, Japan). The PCR conditions were as follows: $95^{\circ} \mathrm{C}$ for $5 \mathrm{~min}$, and then 40 cycles of amplification for $30 \mathrm{~s}$ at $95^{\circ} \mathrm{C}, 45 \mathrm{~s}$ at $60^{\circ} \mathrm{C}$ and $45 \mathrm{~s}$ at $72^{\circ} \mathrm{C}$. Individual gene expression was normalized to $\beta$-actin mRNA. The primer sequences for RT-qPCR of genes are provided in Supplemental Table 1.

\section{Protein extraction and western blot analysis}

HCT116 or RKO cells in 6-well plates were lysed with RIPA Lysis Buffer (Thermo Scientific, Waltham, MA, USA) containing protease inhibitor cocktail (Sigma, St Louis, Missouri, USA) according to the manufacturer's instructions. Protein concentrations were measured with a Pierce BCA protein assay kit (ThermoScientific, New York, USA). Equal amounts of protein were separated by $10 \%$ sodium dodecyl sulfate-polyacrylamide gel electrophoresis (SDS-PAGE) and transferred to a polyvinylidene fluoride (PVDF) membrane (Merck Millipore, Darmstadt, Germany). The antibodies for the western blot analysis in this study were as follows: goat anti-human 4IgB7-H3 (R\&D Systems, \#AF1027), mouse anti-human HK2 (Novus Biologicals, \#NBP1-51643), rabbit anti-human/ mouse STAT3 (CST, \#12640, MA, USA), rabbit antihuman/mouse Phospho-STAT3 (pSTAT3) (CST, \#9145), rabbit anti-human Bcl-2 (abcam, \#ab32124), rabbit antihuman/mouse Bax (abcam, \#ab32503) and mouse antihuman/mouse $\beta$-actin (CST, \#3700). The membranes were then developed with Clarity Western ECL substrate (Bio-Rad) and visualized with a ChemiDoc ${ }^{\mathrm{TM}}$ MP Imaging System (Bio-Rad). 


\section{Proliferation assay and colony formation assay}

Cell viability was assessed using the Cell Counting Kit-8 (CCK8) assay (Dojindo Laboratory, Japan) according to the manufacturer's protocol. For the L-OHP and 5-FU viability assays, 5000 cells/well were seeded in 96-well plates, and then L-OHP $(5 \mu \mathrm{mol} / \mathrm{L})$ or 5 -FU $(10 \mu \mathrm{mol} / \mathrm{L})$ was added (Sigma). Viability was measured after treatment with L-OHP or 5-FU for $48 \mathrm{~h}$.

For the colony formation assay, cells were seeded in 12well plates and then treated with $5 \mu \mathrm{mol} / \mathrm{L} \mathrm{L}-\mathrm{OHP}$. After $2 \mathrm{~h}$, the cells were harvested using trypsin, collected, and reseeded at a density of 2000 cells/well in 12-well plates. Then, the colonies were fixed and stained with $0.1 \%$ crystal violet (Sigma) 14 days later.

\section{Cell apoptosis assay}

Cell apoptosis was measured using an Annexin-V-PE/7AAD double staining apoptosis kit (BD, Franklin Lakes, $\mathrm{NJ}$ ) according to the manufacturer's instructions. Cells were analyzed by flow cytometry (Beckman Coulter, CA, USA). Annexin-V+/7-AAD- cells and Annexin-V+/7$\mathrm{AAD}+$ cells were the apoptotic cells.

\section{Glucose consumption and lactate production assay}

Glucose consumption and lactate production were tested using a Glucose Assay kit (Sigma) and a Lactate Assay kit (Sigma), respectively, as described previously, respectively ${ }^{50}$. The values were normalized to total protein concentration (Pierce BCA protein assay kit, ThermoScientific).

\section{Xenograft tumor}

All manipulations involving live mice were performed in accordance with currently prescribed guidelines and under a protocol approved by the Institutional Animal Care and Use Committee at Soochow University (Suzhou, China). Six-eight-weeks-old female BALB/c athymic nude mice were obtained from the Experimental Animal Center of the Chinese Academy of Medicine Sciences of Soochow University and maintained in a specific pathogen-free environment. For xenograft experiments, B7-H3-overexpressing HCT116 cells $\left(5 \times 10^{6}\right)$ were harvested, resuspended in $150 \mu \mathrm{l}$ of phosphate buffered solution (PBS) (Beyotime, Shanghai, China) and injected subcutaneously into the right flank of each mouse. Tumor volume (mean $\pm \mathrm{sem} ; \mathrm{mm}^{3}$ ) was calculated according to the following equation: $V\left(\mathrm{~mm}^{3}\right)=\mathrm{S}^{2}\left(\mathrm{~mm}^{2}\right) \times \mathrm{L}(\mathrm{mm}) / 2$, where $\mathrm{S}$ and $\mathrm{L}$ are the smallest and the largest perpendicular tumor diameters, respectively ${ }^{51}$.

To determine the resistant effect of B7-H3 on L-OHP in vivo, the mice were randomly divided into the following groups: empty vector-HCT116 (HCT116-EV), B7-H3overexpression HCT116 (HCT116-B7-H3), HCT116-EVL-OHP, and HCT116-B7-H3-L-OHP. The mice in the HCT116-EV-L-OHP and HCT116-B7-H3-L-OHP groups were intraperitoneally injected with L-OHP $(5 \mathrm{mg} / \mathrm{kg})$ twice a week for 2 weeks. The mice in the HCT116-EV and HCT116-B7-H3 groups received vehicle control. LOHP treatment began on day 6 . To investigate whether the resistant effect of B7-H3 was based on HK2 or aerobic glycolysis, $500 \mathrm{mg} / \mathrm{kg}$ 2-DG (Sigma) or vehicle control was given intraperitoneally into mice in the HCT116-B7-H3 group mice one day before of intraperitoneal injection of L-OHP $(5 \mathrm{mg} / \mathrm{kg})$. L-OHP treatment began on day 6 .

\section{Tissue specimens}

Paraffin blocks of 126 CRC specimens from patients were obtained from the First Affiliated Hospital of Soochow University (Suzhou, China); the specimens contained CRC tissues and adjacent normal tissues and were identified as CRC under hematoxylin and eosin (H\&E) staining. Detailed clinicopathological information is provided in Supplemental Table 2. Ethics approval was obtained from the Institutional Review Board of Soochow University. Informed consent was also obtained from patients for experimentation.

\section{Immunohistochemistry}

Immunohistochemistry (IHC) was performed as previously described ${ }^{6}$. Briefly, sections from paraffin embedded tissues were incubated with goat anti-human 4IgB7-H3 antibody (R\&D Systems, \#AF1027) or mouse anti-human HK2 antibody (Novus Biologicals, \#NBP151643) overnight at $4{ }^{\circ} \mathrm{C}$. This step was followed by staining ( $45 \mathrm{~min}$ at room temperature) with the corresponding HRP-labeled rabbit anti-goat secondary antibody or goat anti-mouse secondary antibody (Invitrogen). Next, the sections were visualized by staining with 3,3'diaminobenzidine (Biocare Medical, California, USA) and counterstaining with hematoxylin (Sigma).

All sections were then reviewed blindly by two experienced pathologists (Dr. Cao and Dr. Zhan). The scoring criteria for B7-H3 and HK2 immunostaining were based on clinical data and adopting the semiquantitative immunoreactive score (IRS) system ${ }^{52}$. Briefly, category A (intensity of immunostaining) was scored using the following criteria: 0 , negative; 1 , weak; 2 , moderate; 3 , strong. Category B (percentage of immunoreactive cells) was scored using the following criteria: $1,(0-25 \%)$; 2, (26-50\%); 3, (51-75\%); and 4, (76-100\%). Final scores were calculated by multiplying the scores of categories A and $B$ in the same section; the scores ranged from 0 to 12 .

\section{Statistical analysis}

The results are expressed as the mean \pm standard error (mean \pm SEM). The differences between groups were analyzed by two-tailed unpaired Student's $t$ test. In addition, a paired Student's $t$ test was used to analyze the replicated experiments. A value of $P<0.05$ was considered significant. 


\section{Acknowledgements}

This work was supported by the Major International (Regional) Joint Research Project (No. 31320103918), the National Natural Science Foundation of China (No.81802843, No.81672372, No. 81874163, No. 81372276 and No. 31500718), the Colleges and Universities Natural Science Research Project of Jiangsu Province (18KJB320023 and 17KJA310004), Suzhou Science \& Technology plan project (SS201631) and the Defense Basic Research Projects.

\section{Author details}

'Jiangsu Institute of Clinical Immunology, The First Affiliated Hospital of Soochow University, 708 Renmin Road, Suzhou, China. ${ }^{2}$ Jiangsu Key Laboratory of Clinical Immunology, Soochow University, 708 Renmin Road, Suzhou, China. ${ }_{3}^{3}$ Jiangsu Key Laboratory of Gastrointestinal tumor Immunology, The First Affiliated Hospital of Soochow University, 708 Renmin Road, Suzhou, China. ${ }^{4}$ Department of Gastroenterology, The First Affiliated Hospital of Soochow University, 188 Shizi Road, Suzhou, China. ${ }^{5}$ Institute of Paediatric Research, Affiliated Children's Hospital of Soochow University, 92 Zhongnan Road, Suzhou, China. ${ }^{6}$ Department of Immunology, School of Medicine, University of Pittsburgh, EBST E1047, 200 Lothrop Street, Pittsburgh, PA, USA

\section{Conflict of interest}

The authors declare that they have no conflict of interest.

\section{Publisher's note}

Springer Nature remains neutral with regard to jurisdictional claims in published maps and institutional affiliations.

Supplementary Information accompanies this paper at (https://doi.org/ 10.1038/s41419-019-1549-6).

Received: 21 January 2019 Revised: 22 March 2019 Accepted: 25 March 2019

Published online: 05 April 2019

\section{References}

1. Baena, R. \& Salinas, P. Diet and colorectal cancer. Maturitas 80, 258-264 (2015).

2. Meyerhardt, J. A. \& Mayer, R. J. Systemic therapy for colorectal cancer. N. Engl. J. Med. 352, 476-487 (2005).

3. Chapoval, A. I. et al. B7-H3: a costimulatory molecule for T cell activation and IFN-gamma production. Nat. Immunol. 2, 269 (2001).

4. Sun, M. et al. Characterization of mouse and human B7-H3 genes. J. Immunol. 168, 6294-6297 (2002).

5. Ni, L. \& Dong, C. New B7 family checkpoints in human cancers. Mol. Cancer Ther. 16, 1203-1211 (2017)

6. Bin, Z. et al. Overexpression of B7-H3 in CD133+ colorectal cancer cells is associated with cancer progression and survival in human patients. J. Surg. Res. 188, 396-403 (2014)

7. Ingebrigtsen, V. A. et al. B7-H3 expression in colorectal cancer: nuclear localization strongly predicts poor outcome in colon cancer. Int. J. Cancer 131, 2528-2536 (2012).

8. Jiang, B. et al. The co-stimulatory molecule B7-H3 promotes the epithelialmesenchymal transition in colorectal cancer. Oncotarget 7, 31755-31771 (2016).

9. Seaman S. et al. Eradication of tumors through simultaneous ablation of CD276/B7-H3-positive tumor cells and tumor vasculature. Cancer Cell 31, 501-515 e508 (2017).

10. Zhang, P., Chen, Z., Ning, K., Jin, J. \& Han, X. Inhibition of B7-H3 reverses oxaliplatin resistance in human colorectal cancer cells. Biochem. Biophys. Res. Commun. 490, 1132-1138 (2017).

11. Sun, Z. Z. et al. B7-H3 upregulates BRCC3 expression, antagonizing DNA damage caused by 5-Fu. Oncol. Rep. 36, 231-238 (2016).

12. Hanahan, D. \& Weinberg, R. A. Hallmarks of cancer: the next generation. Cell 144, 646-674 (2011).

13. Heiden, M. G. V., Cantley, L. C. \& Thompson, C. B. Understanding the Warburg effect: the metabolic requirements of cell proliferation. Science 324, 1029-1033 (2009).
14. Ngo, H., Tortorella, S. M., Ververis, K. \& Karagiannis, T. C. The Warburg effect: molecular aspects and therapeutic possibilities. Mol. Biol. Rep. 42, 825-834 (2015).

15. Yeung, S. C. J., Pan, J. \& Lee, M. H. Roles ofp53, Myc and HIF-1 in regulating glycolysis - the seventh hallmark of cancer. Cell. Mol. Life Sci. 65, 3981-3999 (2008).

16. Kim, J. W., Tchernyshyov, I., Semenza, G. L. \& Dang, C. V. HIF-1-mediated expression of pyruvate dehydrogenase kinase: a metabolic switch required for cellular adaptation to hypoxia. Cell. Metab. 3, 177-185 (2006).

17. Dang, C. V. MYC on the path to cancer. Cell 149, 22-35 (2012).

18. Ward, P. S. \& Thompson, C. B. Metabolic reprogramming: a cancer hallmark even warburg did not anticipate. Cancer Cell. 21, 297-308 (2012).

19. Pan X., Feng J., Zhang G. Sa1691 MiR-181b-1 Controls Glycolysis As STAT3 activator in colon cancer cells. Gastroenterology 144, S-284 (2013).

20. Pate, K. T. et al. Wnt signaling directs a metabolic program of glycolysis and angiogenesis in colon cancer. EMBO J. 33, 1454-1473 (2014).

21. $\mathrm{Ma}$, X. et al. Lin28/let-7 axis regulates aerobic glycolysis and cancer progression via PDK1 Nat. Commun. 5, 5212 (2014).

22. Lim, S. et al. Immunoregulatory protein B7-H3 Reprograms glucose metabolism in cancer cells by ROS-mediated stabilization of HIF1alpha. Cancer Res. $\mathbf{7 6}$ 2231-2242 (2016).

23. Nunesxavier, C. E. et al. Decreased expression of B7-H3 reduces the glycolytic capacity and sensitizes breast cancer cells to AKT/mTOR inhibitors. Oncotarget 7, 6891-6901 (2016).

24. Li, M. et al. STAT3 regulates glycolysis via targeting hexokinase 2 in hepatocellular carcinoma cells. Oncotarget 8, 24777-24784 (2017).

25. Liu, Y. H., Wei, X., Hu, G. Q. \& Wang, T. X. Quinolone-indolone conjugate induces apoptosis by inhibiting the EGFR-STAT3-HK2 pathway in human cancer cells. Mol. Med. Rep. 12, 2749-2756 (2015).

26. Liu, F., Zhang, T., Zou, S., Jiang, B. \& Hua, D. B7H3 promotes cell migration and invasion through the Jak2/Stat3/MMP9 signaling pathway in colorectal cancer. Mol. Med. Rep. 12, 5455-5460 (2015).

27. Zhang, T., Jiang, B., Zou, S. T., Liu, F. \& Hua, D. Overexpression of B7-H3 augments anti-apoptosis of colorectal cancer cells by Jak2-STAT3. World J. Gastroenterol. 21, 1804-1813 (2015).

28. Bhattacharya, B., Omar, M. F. M. \& Soong, R. The Warburg effect and drug resistance. Br. J. Pharmacol. 173, 970-979 (2016).

29. Wolf, A. et al. Hexokinase 2 is a key mediator of aerobic glycolysis and promotes tumor growth in human glioblastoma multiforme. J. Exp. Med. 208, 313-326 (2011)

30. Ingebrigtsen, V. A. et al. B7-H3 expression in colorectal cancer: associations with clinicopathological parameters and patient outcome. BMC. Cancer 14, 602-602 (2014).

31. Papandreou, I., Cairns, R. A., Fontana, L., Lim, A. L. \& Denko, N. C. HIF-1 mediates adaptation to hypoxia by actively downregulating mitochondrial oxygen consumption. Cell. Metab. 3, 187-197 (2006).

32. Gill, K. S. et al. Glycolysis inhibition as a cancer treatment and its role in an antitumour immune response. Biochim. Biophys. Acta 1866, 87-105 (2016).

33. Coelho, R. G. et al. Hexokinase and phosphofructokinase activity and intracellular distribution correlate with aggressiveness and invasiveness of human breast carcinoma. Oncotarget 6, 29375-29387 (2015).

34. Mukherjee, A. et al. Lysophosphatidic acid up-regulates hexokinase II and glycolysis to promote proliferation of ovarian cancer cells. Neoplasia 17, 723-734 (2015).

35. Huang, X. et al. HK2 is a radiation resistant and independent negative prognostic factor for patients with locally advanced cervical squamous cell carcinoma. Int. J. Clin. Exp. Pathol. 8, 4054-4063 (2015).

36. Choi, B., Pae, H., Kim, Y. \& Chung, H. Nitric oxide-mediated cytoprotection of hepatocytes from glucose deprivation-induced cytotoxicity: involvement of heme oxygenase-1. Hepatology 37, 810-823 (2003).

37. Mathupala, S. P., Ko, Y. H. \& Pedersen, P. L. Hexokinase-2 bound to mitochondria: cancer's stygian link to the "Warburg effect" and a pivotal target for effective therapy. Semin. Cancer Biol. 19, 17-24 (2009).

38. Guo, W. et al. MiR-199a-5p is negatively associated with malignancies and regulates glycolysis and lactate production by targeting hexokinase 2 in liver cancer. Hepatology 62, 1132-1144 (2015).

39. LV, X., Yao, L., Zhang, J., Han, P. \& Li, C. Inhibition of microRNA-155 sensitizes lung cancer cells to irradiation via suppression of HK2-modulated glucose metabolism. Mol. Med. Rep. 14, 1332-1338 (2016).

40. Gregersen, L. H. et al. MicroRNA-143 down-regulates Hexokinase 2 in colon cancer cells. BMC. Cancer 12, 232-232 (2012). 
41. Zhuo, B. et al. PI3K/Akt signaling mediated Hexokinase-2 expression inhibits cell apoptosis and promotes tumor growth in pediatric osteosarcoma. Biochem. Biophys. Res. Commun. 464, 401-406 (2015).

42. Li, Z., Li, X., Wu, S., Xue, M. \& Chen, W. Long non-coding RNA UCA1 promotes glycolysis by upregulating hexokinase 2 through the mTOR-STAT3/microRNA143 pathway. Cancer Sci. 105, 951-955 (2014).

43. Park, S. H. et al. SIRT2 is a tumor suppressor that connects aging, acetylome, cell cycle signaling, and carcinogenesis. Transl. Cancer Res. 1, 15-21 (2012).

44. Zhang, W. et al. B7-H3 silencing inhibits tumor progression of mantle cell lymphoma and enhances chemosensitivity. Int. J. Oncol. 46, 2562-2572 (2015).

45. Zhang, W. et al. B7-H3 silencing by RNAi inhibits tumor progression and enhances chemosensitivity in U937 cells. Onco. Targets Ther. 8, 1721-1733 (2015).

46. He, C. et al. Astragaloside IV enhances cisplatin chemosensitivity in non-small cell lung cancer cells through inhibition of B7-H3. Cell. Physiol. Biochem. 40, 1221-1229 (2016).
47. Ulanovskaya, O. A., Cui, J., Kron, S. J. \& Kozmin, S. A. A pairwise chemical genetic screen identifies new inhibitors of glucose transport. Chem. Biol. 18, 222-230 (2011).

48. Smith, T. A. D. Influence of chemoresistance and p53 status on fluoro-2-deoxyd-glucose incorporation in cancer. Nucl. Med. Biol. 37, 51-55 (2010).

49. Sayed, S. M. E. et al. The promising anticancer drug 3-bromopyruvate is metabolized through glutathione conjugation which affects chemoresistance and clinical practice: an evidence-based view. Med. Hypotheses 100, 67-77 (2017).

50. Kawauchi, K., Araki, K., Tobiume, K. \& Tanaka, N. p53 regulates glucose metabolism through an IKK-NF-kappaB pathway and inhibits cell transformation. Nat. Cell Biol. 10, 611-618 (2008).

51. Sun, D. et al. MicroRNA-31 activates the RAS pathway and functions as an oncogenic MicroRNA in human colorectal cancer by repressing RAS p21 GTPase activating protein 1 (RASA1). J. Biol. Chem. 288, 9508-9518 (2013).

52. Liu, M. et al. Heterochromatin protein HP1 1 promotes colorectal cancer progression and is regulated by miR-30a. Cancer Res. 75, 4593-4604 (2015). 